\title{
Fuzzy modeling of a hybrid solar dryer: experimental validation
}

\author{
Ahmed Zoukit \\ LSET, Applied Physics Department, FST, Cadi Ayyad University, Marrakesh, Morocco, \\ ahmed1991zoukit@gmail.com, ORCID: 0000-0001-5406-6038 \\ Hicham El Ferouali \\ LSET, Applied Physics Department, FST, Cadi Ayyad University, Marrakesh, Morocco, \\ hichamelferouali@gmail.com. ORCID: 0000-0001-7541-4193
}

Issam Salhi

LSET, Applied Physics Department, FST, Cadi Ayyad University, Marrakesh, Morocco, isalhi@yahoo.fr. ORCID: 0000-0001-8399-7827

\section{Said Doubabi}

LSET, Applied Physics Department, FST, Cadi Ayyad University, Marrakesh, Morocco, s.doubabi@ac.ma. ORCID: 0000-0001-9947-5206

\section{Naji Abdenouri}

LSET, Applied Physics Department, FST, Cadi Ayyad University, Marrakesh, Morocco, n.abdenouri@uca.ma. ORCID: 0000-0002-9399-5435

\section{Arrived: 06.09 .2018 Accepted: 17.12.2018 Published: 31.03 .2019}

\begin{abstract}
A Takagi Sugeno fuzzy (TSF) modeling of an indirect hybrid solar-electrical dryer operated in forced convection $(0.027 \mathrm{~kg} / \mathrm{s})$ was developed. The hybrid dryer was considered as a nonlinear and uncertain system where the operating point varies with weather conditions and airflow. The proposed TSF model was used to predict the drying temperature in no load conditions. Different experimental measurements were set up and used for evaluating the reliability of this model. At first and before applying this method to hybrid solar-electrical dryer, the TSF modeling was tested on solar mode and electrical mode where only one energy source was considered in each mode. The proposed model was experimentally validated in two main modes of the dryer operation: solar mode and electrical mode. The predicted behavior was closed to the experimental data with Root Mean Square Error (RMSE) of 2.34 and 2.21 in solar mode and electrical mode, respectively. The obtained predicted behavior confirms the pertinence of the identified model. The TSF model of the dryer leads to predict the drying temperature instantly with a huge reduction in simulation time in comparison with other modeling techniques. Thus, it is useful for synthesizing a control system of the drying parameters.
\end{abstract}

Keywords: Solar energy; Hybrid dryer; Drying temperature; Takagi Sugeno Fuzzy modeling

Cite this paper as.

Zoukit, A., El Ferouali, H., Salhi, I., Doubabi, S., Abdenouri, N. Fuzzy modeling of a hybrid solar dryer: experimental validation. Journal of Energy Systems, 2019; 3(1): 1-12, DOI: 10.30521/jes.457645

(C) 2019 Published by peer-reviewed open access scientific journal, JES at DergiPark (www.dergipark.gov.tr/jes)

\begin{tabular}{r|l}
\multicolumn{1}{c|}{ Nomenclature } & \\
\hline$T_{c h}$ & Drying chamber temperature $\left[{ }^{\circ} \mathrm{C}\right]$ \\
$T_{a}$ & Ambient temperature $\left[{ }^{\circ} \mathrm{C}\right]$ \\
$G$ & Solar radiation $\left[\mathrm{W} / \mathrm{m}^{2}\right]$ \\
$P_{g}$ & Gas power $[\mathrm{W}]$ \\
$\dot{m}$ & Airflow $[\mathrm{kg} / \mathrm{s}]$ \\
\hline
\end{tabular}




\section{INTRODUCTION}

Solar energy is an important alternative source of energy. It is relatively preferred to other sources because it is free, abundant, inexhaustible and non-pollutant in nature compared with higher prices and shortage of fossil fuels [1,2].

Solar drying is one of the most potential applications. Farmers and citizen's dry food, fruits, seeds, medicinal plants and vegetables by spreading them in thin layers, on mats, paved ground or in the fields, thus exposing them to the sun. This process is not very hygienic, it depends on weather conditions and there is a risk of deterioration [3]. Some of the problems associated with open-air drying could be solved through the use of indirect solar dryers which can reduce crop losses and improve the quality of dried products [4]. According to the literature survey, different kinds of indirect solar dryers were constructed and investigated $[5,6]$. In the last configuration, the air was heated by a solar collector connected to a drying chamber. The products remain in shade and isolated from the sunlight and ambient air. The process occurred by the exchange of water from the wet products to the hot air.

The temperature inside the drying chamber is the most important parameter that affects directly the drying rate. Nevertheless, the fluctuating of weather conditions and the intermittency of solar energy highly affect the temperature threshold of drying air. Therefore, the quality of the dried products is strongly affected. Hence, solar dryers are still limited and could not be effective without the presence of another energy source and without control of all drying parameters (temperature, humidity and airflow).

Hybrid solar-electrical dryers are operating with a solar energy and electrical power as a secondary energy source. According to literature survey, many works $[7,8,9,10]$ used heat resistors inside the drying cells in order to maintain the drying temperature in a suitable range with high efficiency of heat energy exchange.

For efficient controlling system, predicting values of the output parameters related to input conditions is necessary [11]. It is represented by a predictive model of the system. The prediction of drying temperature can be established using several models based on heat transfer, movement and mass equation. These models require a hug computational time compared with the settling time of the dryer.

In previous works, solar dryers were modeled using various techniques [12]: artificial neural networks, Adaptive Neuron-Fuzzy Inference System (ANFIS), FUZZY, thermal modeling, mathematical modeling and drying kinetics model, etc. But, there is no literature found in modeling hybrid solar dryers by using model which can provide predictive output in enough less time to permit the complete control of temperature inside dryer.

Fuzzy logic modeling is based on input and output data, applied on nonlinear multi-inputs and multioutputs systems $[13,14]$. The strength of fuzzy logic is the accuracy of output prediction even if the system is nonlinear and the input parameters are floating. Fuzzy modeling and fuzzy sets are widely used for predictions and estimations of floating and nonlinear parameters as reported in [15-17]. The behavior of solar dryer remains nonlinear in widely operated cases.

In all previous works, Fuzzy model was used only for modeling solar dryers supplied by solar irradiation without secondary energy source and only for the specific product drying [18-21]. This technique was used to predict the drying behavior taking into account different weather conditions, mainly ambient temperature, solar radiation, and relative humidity [18]. A fuzzy model was also developed to determine and calculate the temperature and moisture of jiggery during the evaporation of water content in a greenhouse system [19]. On the other hand, prediction models in no load conditions would provide thermal behavior of the dryer. Based on this, suitable range of products of interest will be specified for 
the effective drying. In order to predict the greenhouse air temperature and relative humidity, an ANFIS prediction model of a greenhouse dryer in no load conditions was proposed in [20]. Authors in [21] proposed no load performances index for various solar dryers.

The main contribution of this paper is to perform an alternative method for modeling a hybrid solarelectrical dryer in no load conditions by using Takagi-Sugeno fuzzy (TSF) system. This method has never been explored for modeling hybrid dryers. The nonlinear system is linearized into different operating points. Then, linear models corresponding to every operating point are established. The fuzzy model is described by a family of fuzzy IF-THEN rules where each one represents a linear input-output relation of the system. The global fuzzy model of the studied dryer was achieved by smoothly blending these linear models together through the fuzzy membership functions in order to give the overall nonlinear behavior of the dryer.

The principal objective of this work is to develop a TSF model of hybrid solar-electrical dryer in order to predict accurately and quickly its thermal behavior in forced convection and in two operating modes: solar mode (energy was only provided by solar energy) and electrical mode (energy was only provided by electrical power). Fuzzy rules of the system were obtained based on experimental measurements of drying temperature at various solar radiations and electrical powers. Some simple linear models for several operating points were identified using Matlab system identification Toolbox (Ident) and were used to build the global TSF model of the studied dryer. Then, the developed model was experimentally validated at various solar radiation, ambient temperatures and electrical powers.

\section{DESCRIPTION of EXPERIMENTAL SETUP}

The solar dryer consists mainly of a solar collector where the air used in the drying process was heated and a drying chamber where the material to be dried was spread in tray, electrical fan allowing the choice of the desired airflow, resistance heater $(4 \mathrm{~kW}$ : accuracy $+/-2 \%)$ and an analogue power controller. The solar collector is a finned absorber (Length $=2 \mathrm{~m}$ and Width $=1 \mathrm{~m}$ ) made from aluminum. The top side of the absorber is painted with matt black glycerophtalic lacquer (absorptivity $=0.95$ and emissivity $=$ 0.8 ). The number of fins is 24 and they are made from aluminum with $1 \mathrm{~mm}$ thickness. The top losses are minimized by placing a glass cover of $3 \mathrm{~mm}$ thickness with a distance of $50 \mathrm{~mm}$ over the top of the absorber. The collector is stationary and inclined at a tilt angle of $30^{\circ}$ (respect to the latitude of Marrakesh, Morocco) with the horizontal facing south all the duration of experiments. The bottom and sides are isolated with glass wool $40 \mathrm{~mm}$ thick. The solar collector is combined directly to the drying chamber without using any air conducts. The drying chamber was built from a steel structure. The walls were made from steel sheet metal and were isolated by mineral fiber $40 \mathrm{~mm}$ thick. The total capacity of the drying chamber is four mesh trays with a drying area of $0.94 \mathrm{~m}^{2}$ (Figure 1).

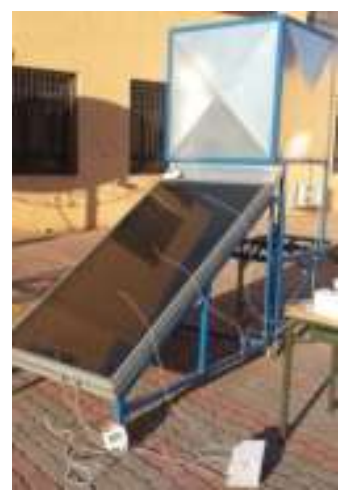

Figure 1. Photo of an indirect hybrid solar-electrical dryer. 
Figure 2 shows a schematic layout of the hybrid indirect solar-electrical dryer.

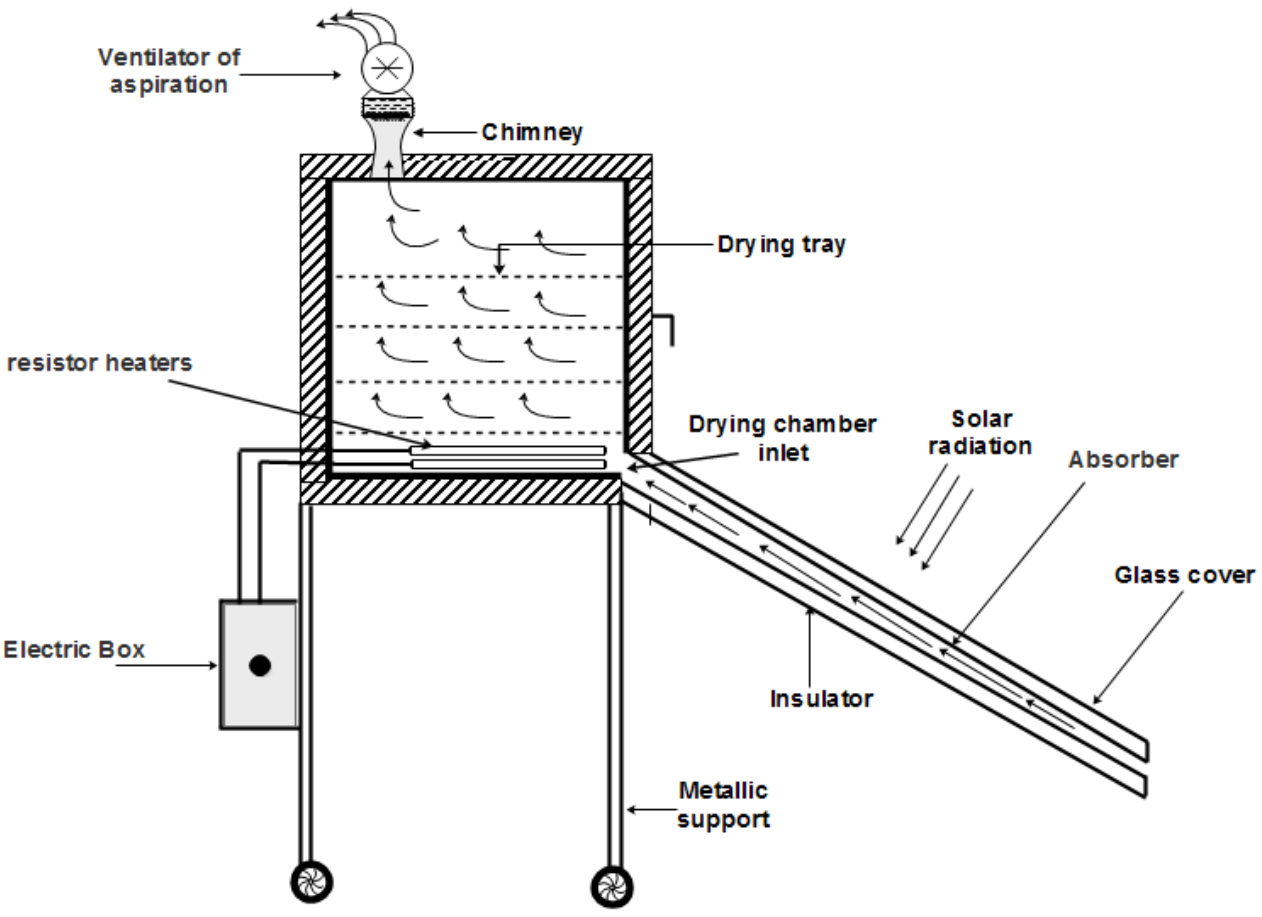

Figure 2. Bloc diagram of the hybrid solar dryer.

\section{MATERIALS and METHODS}

The dryer was unloaded because the investigations in no load conditions provide consistent results as they are not influenced by the type, the composition and the moisture content of food products. The experiments were conducted during several days starting at 11:00 am and finished at 13:30 pm. During this period, solar radiation and ambient temperature can be assumed constants. The dryer was placed in an unshaded and clear area during the whole duration of experiments. A fan was fixed in the chimney of the dryer to ensure an even distribution of air and also to exhaust the humidity of the product to the surrounding. In solar drying process, the drying air temperature changes with the magnitude of solar radiation. Thus, the auxiliary electric heater was used to adjust the drying air temperature. The preliminary heated drying air by solar radiation, arrived to the inlet of cabinet dryer will be heated by electrical resistance. The power of the electrical resistance was varied using an analogue power controller (037N0057, Danfoss). The drying air is then aspired by the exhaust fan of $10 \mathrm{~cm}$ diameter (Orion 12HBVXC model, 43W power input, running at $5000 \mathrm{rpm}$ and volumic flow at $0.107 \mathrm{~m}^{3} / \mathrm{s}$ ). The fan was controlled by an Arduino Uno for maintaining an air flow of $0.027 \mathrm{~kg} / \mathrm{s}$. Temperature, humidity and solar radiation were recorded every $10 \mathrm{~min}$. Temperature was measured and recorded at different locations inside the drying chamber (bottom, middle and the top) using temperature sensors type (TM$110 \mathrm{pt} 100,0.5^{\circ} \mathrm{C}$ accuracy). Hygrometer sensors (HM-110, $0.5 \%$ accuracy) were employed for measuring the ambient relative humidity. Incident global solar radiation was measured during the experiments by a Keep and Zonnen pyranometer with a sensitivity of $14.6910^{-3}$. Velocity of drying air was measured using an anemometer (Kimo model CTV-210-BOS, $0.03 \mathrm{~m} / \mathrm{s}$ accuracy) at the exit of the exhaust fan. 


\section{NON LINEARITY OF THE HYBRID DRYER}

Experimental measurements of the temperature profile inside the drying chamber at three planes; top, middle and bottom were conducted in forced convection. It is noticed that there is no significant differences in average temperature (maximum difference of $1.4^{\circ} \mathrm{C}$ ) at all planes due to the air vortex inside the drying chamber. However, only the average temperature of the drying chamber was considered for modeling.

The hybrid dryer is a complex nonlinear system. In order to figure out the nonlinear relationship of the dryer's input/output and to investigate its overall behavior, static characteristics of the dryer operated in solar mode and electrical mode were established based on experimental studies. The static characteristics lead also to easily identify the linear zones and the operating points of the dryer.

Static characteristics were built measuring the drying chamber temperature in steady state on the basis of solar radiation and electrical power of the resistance heater. From the conducted tests for different solar radiations (solar radiations and ambient temperatures are considered approximately constant from 11:00 am to $13: 30 \mathrm{pm}$, for each day), the steady state was reached after two hours and a half (at about $13: 30 \mathrm{pm})$.

Figure 3 depicts the static characteristics of the dryer operated in solar mode (Figure 3a) and electrical mode (Figure $3 b$ ). The plots clearly show the strong static non linearity of the dryer by appearance of saturation of the output for high solar radiations. To overcome this problem, the static characteristics of the dryer were divided into linear zones numbered $(1,2,3,4)$ and $\left(1^{\prime}, 2^{\prime}, 3^{\prime}, 4^{\prime}\right)$ corresponding to different weather conditions (windy day, cloudy day, partly cloudy day and clear day) and electrical powers (low power, medium power, high power and very high power). Several operating points were singled out in the linear zones and linear models corresponding to each operating point were identified. A TSF model was developed to ensure the connection between the linear zones by combining the linear models to give the overall nonlinear behavior of the studied hybrid dryer.
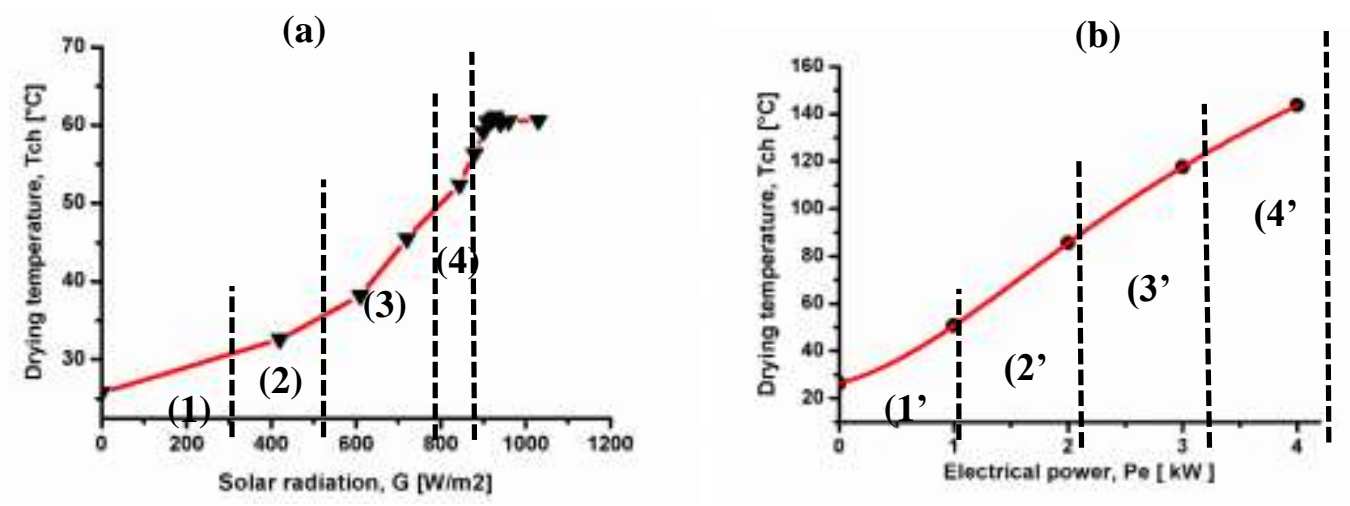

Figure 3. Static characteristics of the hybrid dryer, (a) solar mode; (b) electrical mode.

\section{RESULTS AND DISCUSSIONS}

\subsection{Behavior of the dryer operated in solar mode}

In order to identify the linear models corresponding to each operating point of the dryer operated in solar mode, several measurements were carried out in winter season for days December $12^{\text {th }}, 16^{\text {th }}, 2016$ and January $5^{\text {th }}, 18^{\text {th }}, 2017$ at different weather conditions. The dryer remains previously in shade and 
immediately exposed to solar radiation for two hours and a half starting at 11:00 am. The highest air temperature inside the drying chamber was previously noticed between 11:00 am and 13:30 pm.

In clear days, the highest reached drying temperature was $50.2^{\circ} \mathrm{C}$ under a solar radiation of $964.4 \mathrm{~W} / \mathrm{m}^{2}$ for an ambient temperature of $25.6^{\circ} \mathrm{C}$ (Figure 4a) (measurements corresponding to the operating point in the linear zone 4), and $47.2^{\circ} \mathrm{C}$ at a solar radiation of $804.5 \mathrm{~W} / \mathrm{m}^{2}$ (zone 3 ) and $25.1^{\circ} \mathrm{C}$ ambient temperature (Figure $4 \mathrm{~b}$ ). In a cloudy day (Figure $4 \mathrm{c}$ ), the drying chamber temperature reached $42.2^{\circ} \mathrm{C}$, under a solar radiation and ambient temperature of $630.6 \mathrm{~W} / \mathrm{m}^{2}$ and $23.6^{\circ} \mathrm{C}$, respectively (Zone 2).

Experimental tests were also conducted in a period of stormy weather and air temperature inside the drying chamber reached $14.2^{\circ} \mathrm{C}$ at a solar radiation of $101.3 \mathrm{~W} / \mathrm{m}^{2}$ (Zone 1) and an ambient temperature of $12.1^{\circ} \mathrm{C}$ as shown in (Figure $4 \mathrm{~d}$ ).
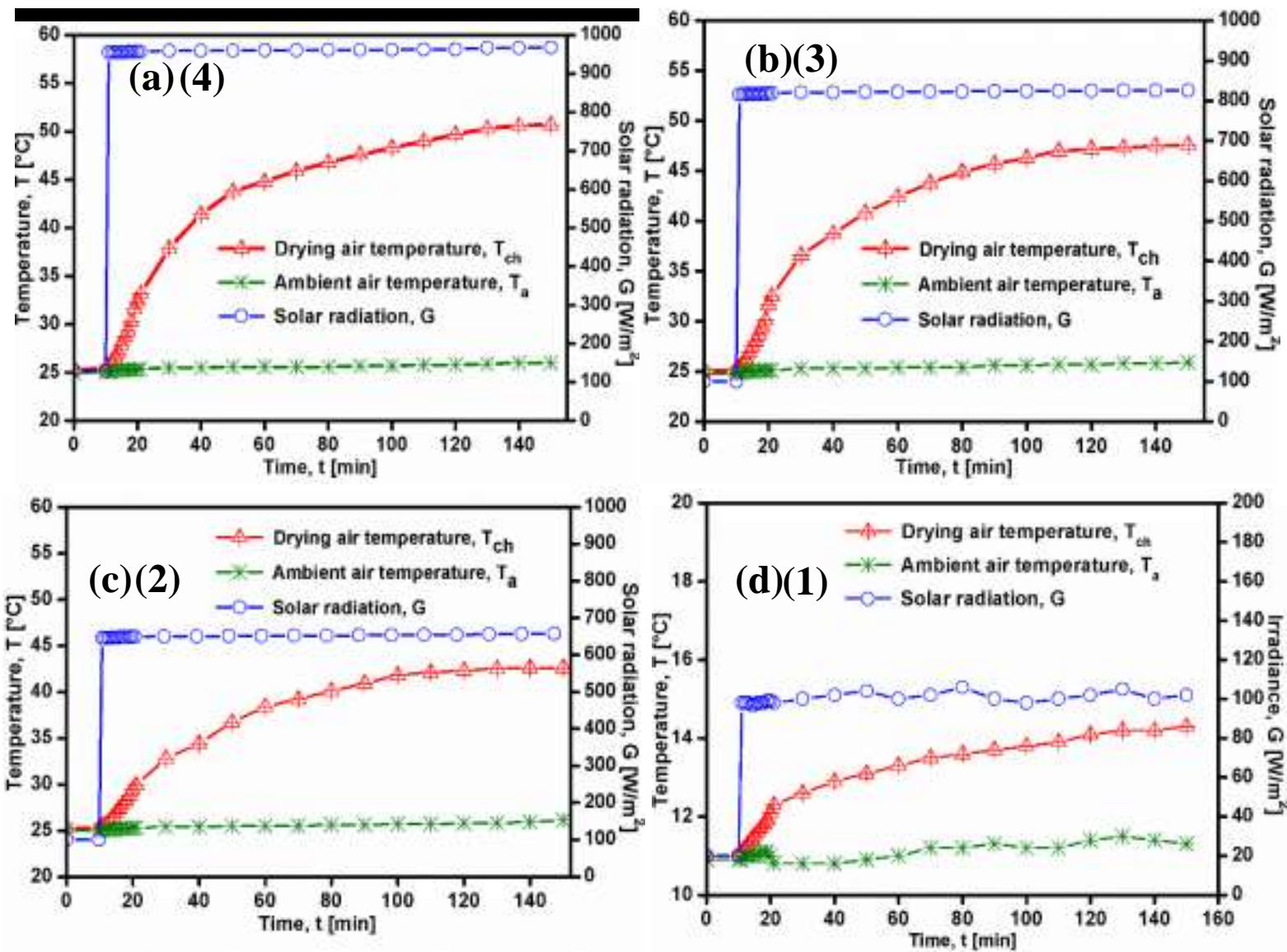

Figure 4. Solar intensity and air temperature variation; (a)(b) clear days, (c) windy day, (d) cloudy day. (1), (2), (3), (4) numbers corresponding to linear zones in static characteristic of the dryer in solar mode.

\subsection{Behavior of the dryer operated in electrical mode}

To identify the linear models corresponding to each operating point of the dryer operated in electrical mode, different measurements were carried out for days January $1^{\text {st }}, 3^{\text {rd }}, 7^{\text {th }}$, and $10^{\text {th }}, 2017$ at different electrical powers. The tests were conducted at night in the absence of solar radiation. The steady state was achieved in one hour of operation.

The drying temperature reached $37.4^{\circ} \mathrm{C}$ at an electrical power of $0.5 \mathrm{~kW}$ and an ambient temperature of $24.6^{\circ} \mathrm{C}$ (Figure 5a (Zone 1')). At an electrical power of $1.5 \mathrm{~kW}$, the temperature reached $69.7^{\circ} \mathrm{C}$ at an ambient temperature of $22.6^{\circ} \mathrm{C}$ (Figure $5 \mathrm{~b}$ (zone $\left.2^{\prime}\right)$ ). The average temperature inside the drying 
chamber reached $100.1^{\circ} \mathrm{C}$ and $128.7^{\circ} \mathrm{C}$ at electrical powers of $2.5 \mathrm{~kW}$ and $3.5 \mathrm{~kW}$ and ambient temperatures of $21.5^{\circ} \mathrm{C}$ and $23.8^{\circ} \mathrm{C}$, respectively as shown in Figures $5 \mathrm{c}, 5 \mathrm{~d}$ (zone $3^{\prime}, 4^{\prime}$ ).
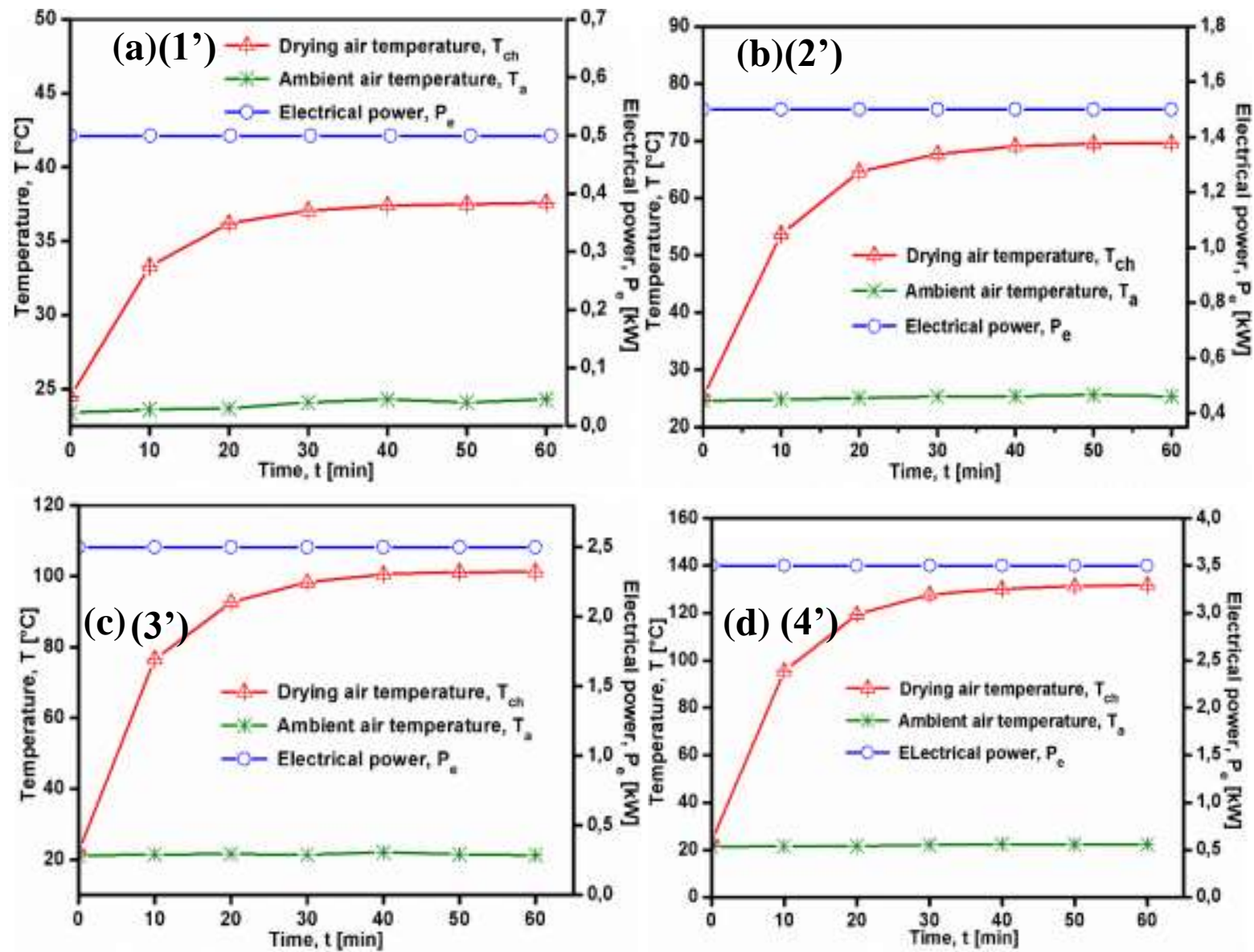

Figure 5. Electrical power and air temperature variation; (a) $0.5 \mathrm{~kW}$ (b) $1.5 \mathrm{~kW},(\mathrm{c}) 2.5 \mathrm{~kW}$, (d) $3.5 \mathrm{~kW}$. (1'), (2'),

(3'), (4') numbers corresponding to linear zones in static characteristic of the dryer in Electrical mode.

\section{HYBRID SOLAR-ELECTRICAL DRYER MODELING}

\subsection{The linear hybrid dryer models}

The hybrid dryer dynamic response can be represented by different linear models depending on the operating points in the three operating modes (solar mode, electrical mode and hybrid mode).

The experimental measurements corresponding to different operating points led to highlight the influence of different inputs (solar radiation, ambient temperature and electrical power) on the behavior of the dryer. In addition, they permit to evaluate the simplest representation (linear models) of the dryer. In order to identify the linear models, the measured inputs and outputs were loaded into system identification Toolbox of Matlab environment using (Ident). This latter provides functions and Simulink blocs for constructing mathematical models based on least squares methods of dynamic systems from measured input/output data. In the three operating modes, the obtained response is similar to that of a second order system (Eq. [1]) with a best fitting of $96.5 \%$ where the parameters change according to each considered operating points. 


$$
F(s)=\frac{a s+b}{s^{2}+c s+d}
$$

The coefficients ( $a, b, c, d)$ are the linear model's parameters. These coefficients were computed using the measured data obtained for different solar radiations and electrical powers. Table 1 gives the identified coefficients of the linear models transfer functions. According to Table 1, the nonlinear evolution of coefficient ( $a, b, c, d)$ can be noticed, which confirms the high nonlinearity of the studied system. It confirms that it is impossible to represent the hybrid solar dryer by a unique second order model for different weather conditions and operating modes. To overcome this issue a TSF system was developed to obtain a global nonlinear model of the studied hybrid dryer.

Table 1. Linear models' parameters based on solar radiation and electrical power.

\begin{tabular}{|c|c|c|c|c|c|}
\hline & & \multicolumn{4}{|c|}{ Coefficients of linear models } \\
\hline & & $\mathrm{a}$ & $\mathrm{b}$ & $\mathrm{c}$ & $\mathrm{d}$ \\
\hline \multirow{4}{*}{ Solar mode } & $964.7 \mathrm{~W} / \mathrm{m}^{2} 25.6^{\circ} \mathrm{C}$ & $1,919.10^{-5}$ & $6,033.10^{-9}$ & 0,001273 & 2,048 \\
\hline & $804.5 \mathrm{~W} / \mathrm{m}^{2} 25.1^{\circ} \mathrm{C}$ & $2,543.10^{-5}$ & $3,3.10^{-8}$ & 0,003218 & $1,165.10^{-6}$ \\
\hline & $463.6 \mathrm{~W} / \mathrm{m}^{2} 23.6^{\circ} \mathrm{C}$ & $2,878 \cdot 10^{-5}$ & $2,226.10^{-8}$ & 0,002761 & $7,518.10^{-7}$ \\
\hline & $251.2 \mathrm{~W} / \mathrm{m}^{2} 20.1^{\circ} \mathrm{C}$ & $3,104 \cdot 10^{-5}$ & $2.053 .10^{-8}$ & 0.002662 & $5.535 .10^{-7}$ \\
\hline \multirow{4}{*}{ Electrical mode } & $0.5 \mathrm{~kW} 24.6^{\circ} \mathrm{C}$ & 34.7 & 24.7 & 2.027 & 0.9491 \\
\hline & $1.5 \mathrm{~kW} 22.6^{\circ} \mathrm{C}$ & 39.7 & 20.68 & 1.82 & 0.65 \\
\hline & $2.5 \mathrm{~kW} 21.5^{\circ} \mathrm{C}$ & 42.5 & 16.3 & 1.53 & 0.48 \\
\hline & $3.5 \mathrm{~kW} 23.8^{\circ} \mathrm{C}$ & 45.2 & 14.2 & 1.21 & 0.41 \\
\hline
\end{tabular}

\subsection{Takagi Sugeno Fuzzy model of the hybrid dryer}

In this paper, a TSF modeling based on linear functions of input variables was considered. It can approximate nonlinear systems with few rules and higher modeling accuracy [22, 23]. This model was used in many works and for many applications, where the linear models are used individually and aids for validating and interpretation of the global model [24, 25]. The TSF model combines all the linear models in order to find a global model of the system able to generate the appropriate output as shown in Figure 6.

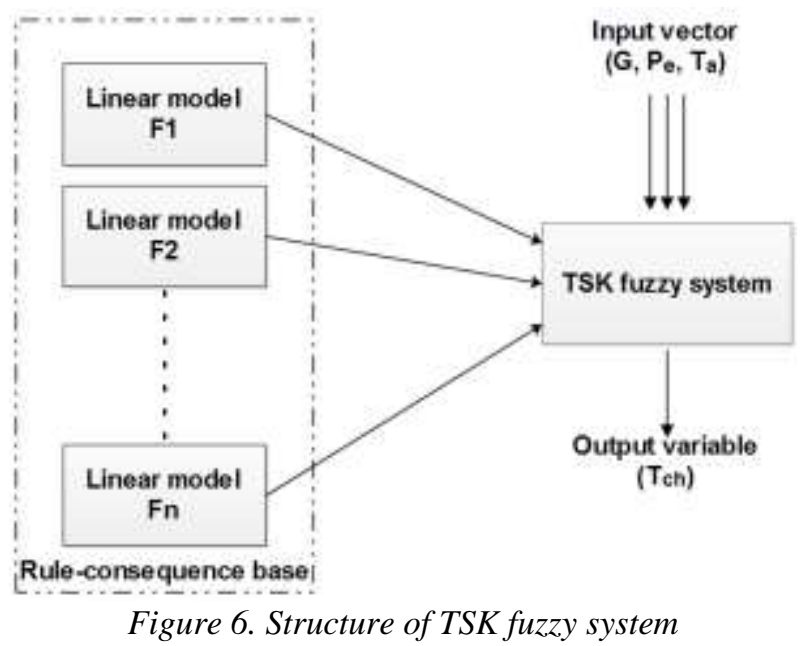

The TSF fuzzy system was built from the input/output and the obtained parameters of linear models. Figure 7 shows the seven membership functions corresponding to the dryer parameter $(G)$ characterizing solar radiation. It can be seen that the generation of membership functions was focused in the high and low solar radiations where the system represents strong nonlinearity. The generated membership functions corresponding to the dryer parameter $\left(P_{e}\right)$ characterizing the electrical power are shown in Figure 8. Figure 9 illustrates the generating membership functions corresponding to ambient temperature $\left(T_{a}\right)$. 


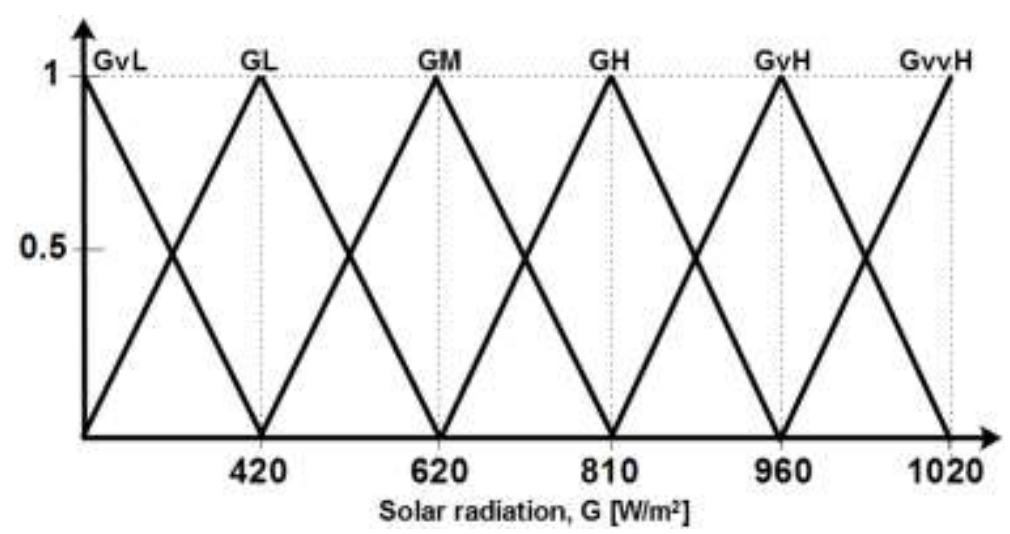

Figure 7. Membership function for solar radiation. $(G v L)$ : Irradiance Very low; $(G L)$ : Irradiance Low; $(G M)$; Irradiance medium; $(G H)$ : Irradiance High; $(G v H)$ : Irradiance very High; $(G v v H)$ : Irradiance very very High.

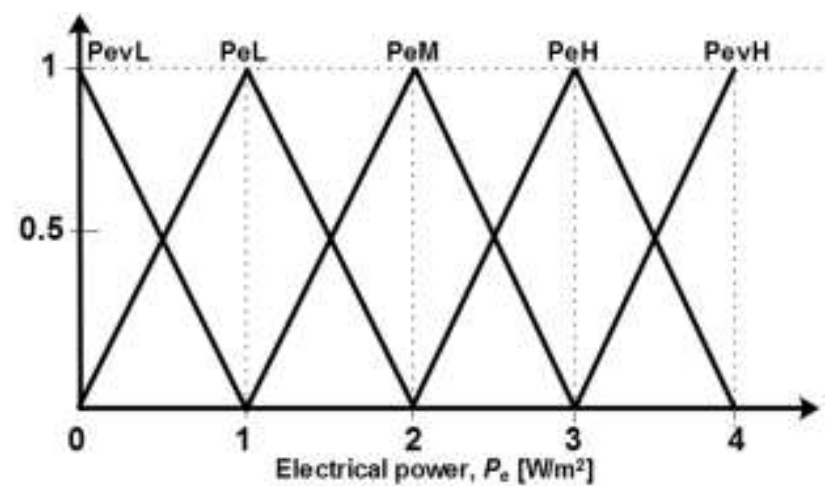

Figure 8. Membership function for electrical power. (PevL): Power Very low; (PeL): Power Low; (PeM); Power medium; (PeH): Power High; (PevH): Power very High.

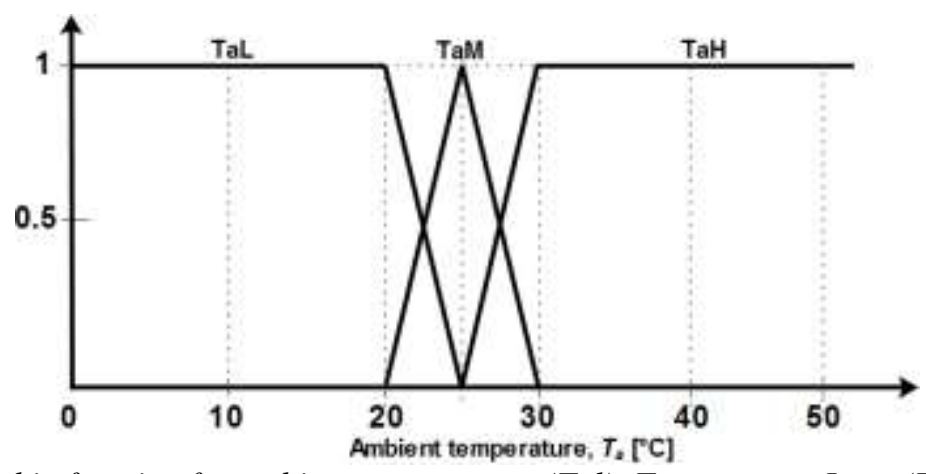

Figure 9. Membership function for ambient temperature. (Tal): Temperature Low; (TaM); Temperature medium; (TaH); Temperature High.

The rule of fuzzy model type Takagi Sugeno are built by the parameters of linear model. Where, each rule represents the parameters of a linear model corresponding to a specific operating point. Thus, the global fuzzy system gives the overall nonlinear behavior of the dryer system.

Using the fuzzy rules, the appropriate TSF model's output (drying temperature) was estimated according to the associated linear models as shown in Figure 10. 


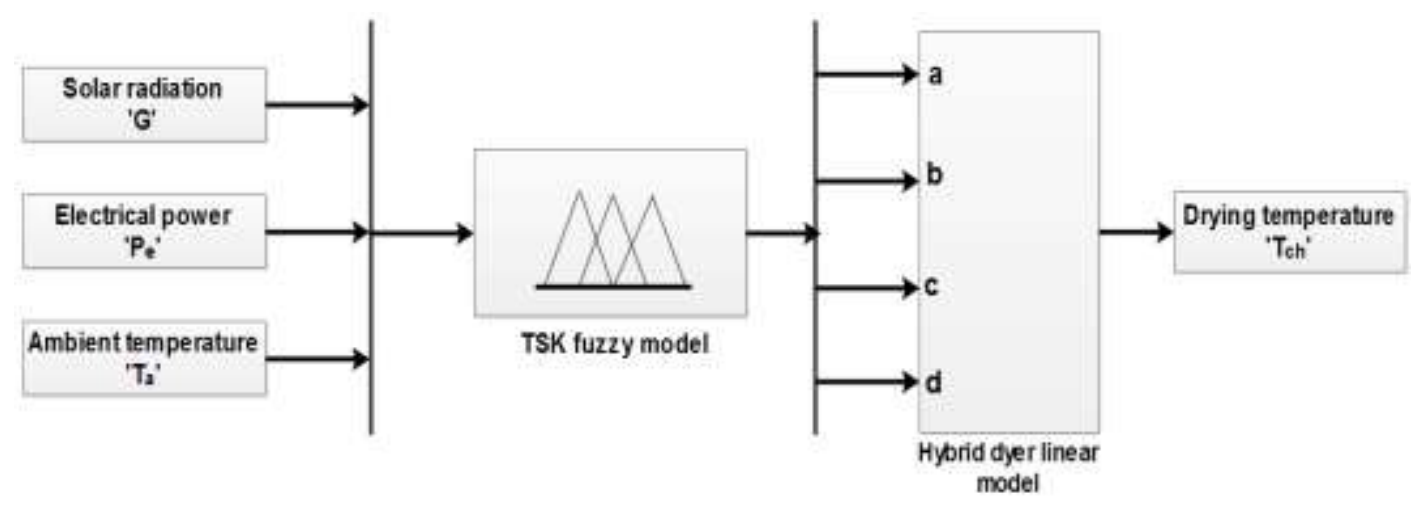

Figure 10. TSK fuzzy model of the hybrid solar-electrical dryer

The rules of the TSF fuzzy models are the following forms $[22,26]$ :

Rule i:

$\mathrm{L}_{\mathrm{i}}$ : if $\quad \mathrm{x}_{1}$ is $\alpha_{\mathrm{i} 1}, \quad \mathrm{x}_{2}$ is $\alpha_{\mathrm{i} 2}, \ldots$, and $\mathrm{x}_{\mathrm{p}}$ is $\alpha_{\mathrm{ip}}$ then $\mathrm{F}_{\mathrm{i}}$.

Where, $L_{i}(i=1,2, \ldots, n)$ is $i$-th fuzzy rule, $x_{j}(j=1,2, \ldots, p)$ is input variable and $F_{i}$ are output parameters of linear models obtained by $\mathrm{L}_{\mathrm{i}}, \alpha_{\mathrm{ij}}(\mathrm{j}=1 \ldots \mathrm{p})$ is the fuzzy set of the variable inputs $\mathrm{j}$.

Given the input $x_{1}^{0}, x_{2}^{0}, \ldots, x_{j}^{0}, x_{m}^{0}$ the global parameters $\mathrm{F}$ can be inferred by a weighted average defuzzification as Eq. [2]

$$
F=\frac{\sum_{i=1}^{n} w_{i} F_{i}}{\sum_{i=1}^{n} w_{i}}
$$

Where the weight $w_{i}$ implies the overall truth value of the premise of the $\mathrm{j}$-th implication for the input, and is calculated as Eq. [3].

$$
\mathrm{w}_{\mathrm{i}}=\prod_{\mathrm{j}=1}^{\mathrm{m}} \mu_{\alpha_{\mathrm{i}}}\left(\mathrm{x}_{\mathrm{j}}\right)
$$

Where $\mu_{\alpha_{i}}\left(x_{j}\right)$ the grade of the membership function of $\mathrm{xj}$ in fuzzy set $\alpha \mathrm{i}$ and it is characterized by a Gaussian function as Eq. [4].

$$
\mu_{\alpha_{i}}\left(x_{j}\right)=\exp \left(-\frac{\left(x_{j}-c_{i}^{j}\right)^{2}}{b_{i}^{j}}\right)
$$

The application of TSF modeling provides an estimation of the dryer behavior for all combinations of solar radiation, ambient temperature and electrical power. This implies that for any weather condition and operating mode (solar mode, electrical mode and hybrid mode) the TSF fuzzy model will predict accurately the drying chamber temperature.

\section{EXPERIMENTAL VALIDATION OF THE HYBRID DRYER TSF MODEL}

The reliability and the performances of the developed model for describing the behavior of the hybrid dryer at three mains operating modes were evaluated by comparing the predicted drying temperature 
with experimentally measured temperature during the operation of the dryer in solar mode, electrical mode and hybrid mode.

Experimental tests were performed in winter season, in clear days, cloudy days and at nights. The dryer was operated in forced convection at an airflow maintained at $0.027 \mathrm{~kg} / \mathrm{s}$.

\subsection{Solar mode}

Experimental measurements were conducted for days $2^{\text {nd }}, 14^{\text {th }}$, January 2017 in a clear and cloudy weather. The average recorded solar radiations and ambient temperatures were $1014 \mathrm{~W} / \mathrm{m}^{2}, 24.5^{\circ} \mathrm{C}$.

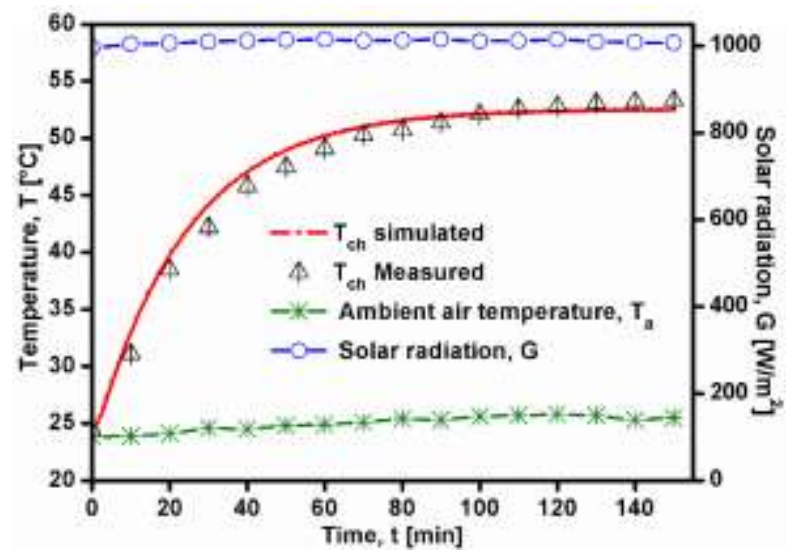

Figure 11. Validation of the constructed TSK model of the dryer operated in solar mode; average solar radiation of $1014.3 \mathrm{~W} / \mathrm{m}^{2}$.

The predicted responses of the model were compared with the experimental ones as shown in Figure 11. It is clearly the measured temperature and the simulated one are in good accordance with an RMSE equal 2.34 .

\subsection{Electrical mode}

In order to test the reliability of the constructed model for dryer operated in electrical mode, experimental measurements were conducted for day $10^{\text {th }}$, February 2017 at night. The considered electrical powers were $2 \mathrm{~kW}$ (Figure 12) with ambient temperatures of $25.6^{\circ} \mathrm{C}$.

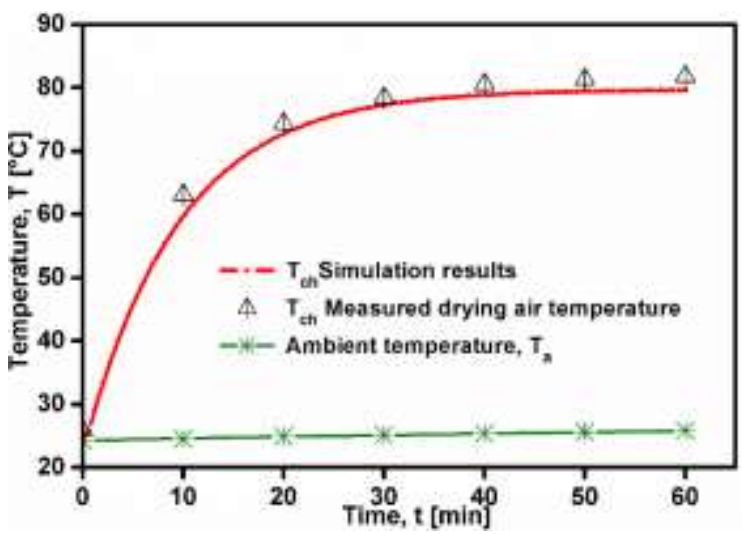

Figure 12. Validation of the constructed TSK model of the dryer operated in electrical mode; average electrical power of $2 \mathrm{~kW}$. 
The predicted response was compared with the experimental ones as shown in Figure 11. It is clearly shown that the fit of drying temperature by the simulated ones is significantly good with an RMSE equal 2.21 .

In Table 2 the values of RMSE of modeling the hybrid dryer operated in solar and electrical mode are presented.

Table 2. Root mean square error (RMSE) of modeling the hybrid dryer.

\begin{tabular}{ll}
\hline Operating mode & RMSE \\
\hline Solar mode & 2.34 \\
Electrical mode & 2.21 \\
\hline
\end{tabular}

\section{CONCLUSION}

In this study, a hybrid solar-electrical dryer modeling operated in forced convection was investigated. A TSF model of the hybrid dryer was developed in order to describe its behavior in three mode of operation (solar mode, electrical mode and hybrid mode) in no load conditions. Static characteristics of the dryer were established to testify the strong nonlinearity of the dryer. The proposed TSF model was explored to predict nonlinear thermal behavior (drying temperature) of the dryer taking into account different solar radiations, ambient temperature, and electrical powers as input parameters. The TSF model was successfully validated through experimental tests with a RMSE of 2.34 and 2.21 for the dryer operated in solar mode and electrical mode, respectively. The proposed TSF model reveals a high prediction ability of the hybrid dryer with a huge reduction in simulation time comparing to other modeling methods. The proposed model will be useful for synthesizing the temperature controller and increasing the efficiency of the dryer to enhance the quality of the dried products. The system could be enhanced by adding a thermal energy storage such as pebbles or rock salts which can help to extend the drying time and also reduce the electrical consumption. Also for the area far from the electricity, the dryer cannot be operated, for this reason a PV models should be used. All this issues will be taken into considerations in future works.

\section{Acknowledgment}

This work was supported by the research institute for solar energy and new energies (IRESEN) as part of the project SSH. The authors are grateful to the IRESEN institute for its cooperation.

\section{REFERENCES}

[1] Frakas, I, Seres, I, Meszaros, C. Analytical and experimental study of a modular solar dryer. Renewable energy 1999; 16: 773-778. DOI: https://doi.org/10.1016/S0960-1481(98)00278-X.

[2] Belloulid, M, O, Hamdi, H, Mandi, L, Ouazzani, N. Solar drying of wastewater sludge: a case study in Marrakesh, Morocco. Environmental Technology (United Kingdom) 2018; 0(0): 1-7. DOI: 10.1080/09593330.2017.1421713.

[3] Chan, Y, Dyah, N, Abdullah, K. Performance of a recirculation type integrated collector drying chamber (ICDC) solar dryer. Energy Procedia 2015; 68: 53-59. DOI: https://doi.org/10.1016/j.egypro.2015.03.232.

[4] Lopez-Vidana, E, C, Mendez-Lagunas, LL, Rodriguez-Ramirez, J. Efficiency of a hybrid solar-gas dryer. Solar energy 2013; 93: 23-31. DOI: https://doi.org/10.1016/j.solener.2013.01.027.

[5] Kumar, A, Singh, R, Prakash, O. Review of global solar drying status. Agric. Eng. Int 2014; 16. 
[6] Prakash, O, Kumar, A, Laguri, V. Performance of modified greenhouse dryer with thermal energy storage. Energy reports 2016; 2: 155-162. DOI: https://doi.org/10.1016/j.egyr.2016.06.003.

[7] Boughali, S, Benmoussa, H, Bouchekima, B, Mennouche, D, Bouguettaia, H, Bechki, D. Crop drying of an indirect active hybrid Solar-Electrical dryer in eastern Algerian Septentrional Sahara. Solar energy 2009; 83: 2223-2232. DOI: https://doi.org/10.1016/j.solener.2009.09.006.

[8] Alejandro, R, Andrea, M, Francisco, C, Pedro, H. Mushroom dehydration in a hybrid solar dryer. Energy conversion and management 2013; 70: 31-39. DOI: https://doi.org/10.1016/j.enconman.2013.01.032.

[9] Augustus Leon, M, Kumar, S. Design and performance evaluation of a solar assisted biomass drying system with thermal storage. Drying technology 2008; 26: 936-947. DOI: https://doi.org/10.1080/07373930802142812.

[10] Ferreira, A, G, Charbel, A, L, T, Peres, R, L, Silva, J, G, Maia, C, B. Experimental analysis of a hybrid dryer. Thermal engineering 2007; 6: 03-07.

[11] Onat, M, Koten, H, Celik, H. Constant Temperature Control with a Fast Transient Response for a Gel Card Incubator, 6th Eur. Conf. Ren. Energy Sys. 25-27 June 2018, Istanbul, Turkey.

[12] Prakash, O, Laguri, V, Pandey, A, Kumar, A. Review on various modelling techniques for the solar dryers. Renew. Sustain. Energy Rev 2016; 62: 396-417. DOI: https://doi.org/10.1016/j.rser.2016.04.028.

[13] Seo, S, Kim, Y, Choi, H, H. Model predictive controller design for boost DC - DC converter using T $-\mathrm{S}$ fuzzy cost function. Int. J. Electron 2017; 104: 838-1853. DOI: https://doi.org/10.1080/00207217.2017.1329945.

[14] Tatli, H, ŞEN, Z. Prediction of Daily Maximum Temperatures Via Fuzzy Sets. Turkish Journal of Engineering and Environmental Sciences 2014; 25 (1): 1-9.

[15] Ibrahim, S. A, Ahmet Sahiner, A, Ibrahim, A. A. Fuzzy Logic Modeling for Prediction of the Nuclear Tracks; Journal of Multidisciplinary Modeling and Optimization 2018; 1: 33-40.

[16] Tütmez, B, Tercan, E. Use of Fuzzy Modeling Approach in Grade Estimation; Scientific Mining Journal 2006; 45: 39-47.

[17] Laouafi, A, Mordjaoui, M, Boukelia T, E. An adaptive neuro-fuzzy inference system-based approach for daily load curve prediction. Journal of energy system 2018; 2(3): 115-126.

[18] Prakash, O, Kumar, A, ANFIS modelling of natural convection greenhouse drying system for jiggery: an experimental validation. Int. J. Sustain. Energy 2014; 33: 316-335. DOI: https://doi.org/10.1080/14786451.2012.724070.

[19] Prakash, O, Kumar, A, Kaviti, AK, Kumar, PV. Prediction of the rate of moisture evaporation from jiggery in greenhouse drying using the fuzzy logic. Heat Transf. Res 2015; 46.

[20] Prakash, O, Kumar, A. ANFIS prediction model of a modified active greenhouse dryer in no-load condition in the month of January. Int. J. Adv.Comput. Res 2013; 3: 220-223.

[21] Singh, S, Kumar, S. Testing method for thermal performance based rating of various solar dryer designs. Solar energy 2012; 86: 87-98. DOI: https://doi.org/10.1016/j.solener.2011.09.009.

[22] Takagi, T, Sugeno, M. Fuzzy identification of systems and its applications to modeling and control. IEEE trans 1985; 15: 116-132. DOI: 10.1109/TSMC.1985.6313399.

[23] Zhu, B, He, CZ, Liatsis, P, Li, XY. A GMDH-based fuzzy modeling approach for constructing TS model. Fuzzy Sets Syst 2012 ; 189 : 19-29. DOI: https://doi.org/10.1016/j.fss.2011.08.004.

[24] Du, H, Zhang, N. Application of evolving Takagi-Sugeno fuzzy model to linear system identification. App. Soft Comput J 2008; 8: 676-686. DOI: 10.1016/j.asoc.2007.05.006 .

[25] Johansen, T, Shorten, R, Murray-Smith, R. On the interpretation and identification of dynamic (TakagiSugeno) fuzzy models. IEEE transaction 2000; 8: 297-313. DOI: 10.1109/91.855918.

[26] El Hamdaoui, A, Salhi, I, Belattar, A, Doubabi, S. Modeling a three-phase micro hydropower plant prototype using Takagi Sugeno fuzzy approach. International journal of hydrogen energy 2017; 42: 575-581. DOI: https://doi.org/10.1016/j.ijhydene.2017.02.167. 